This is the final peer-reviewed accepted manuscript of:

S. V. Suraci, D. Fabiani, X. Colin and S. Roland,

"Chemical and electrical characterization of XLPE cables exposed to radio-thermal aging"

in "2020 IEEE 3rd International Conference on Dielectrics (ICD)", 2020, pp. 57-60

The final published version is available online at:

https://doi.org/10.1109/ICD46958.2020.9341895

Rights / License:

The terms and conditions for the reuse of this version of the manuscript are specified in the publishing policy. For all terms of use and more information see the publisher's website.

This item was downloaded from IRIS Università di Bologna (https://cris.unibo.it/)

When citing, please refer to the published version. 


\section{Chemical and electrical characterization of XLPE cables exposed to radio-thermal aging}

\author{
Simone Vincenzo Suraci* \\ LIMES (Laboratory of Innovative \\ Materials for Electrical Systems) - DEI \\ University of Bologna \\ PIMM, HESAM University, Paris \\ simone.suraci@unibo.it
}

\author{
Davide Fabiani \\ LIMES (Laboratory of Innovative \\ Materials for Electrical Systems) - DEI \\ University of Bologna \\ Bologna, Italy \\ davide.fabiani@unibo.it \\ Sébastien Roland \\ PIMM, Arts et Métiers Institute of \\ Technology, CNRS, CNAM, HESAM \\ University, Paris (France) \\ Sebastien.roland@ensam.eu
}

\author{
Xavier Colin \\ PIMM, Arts et Métiers Institute of \\ Technology, CNRS, CNAM, HESAM \\ University, Paris (France) \\ xavier.colin@ensam.eu
}

\begin{abstract}
The aging of twisted pair cables with silane crosslinked polyethylene insulation has been examined by means of both chemical and electrical techniques. Radio-chemical aging has been performed through three different dose rates in order to evaluate the contribution of the dose rate on the changing of the analyzed properties with aging. Correlation between the different techniques has been performed and the antioxidant contents has been successfully linked to the variation of the dielectric properties.
\end{abstract}

Keywords-XLPE aging, Gamma radiation, low-voltage cables, dielectric spectroscopy, chemical properties, electrical properties.

\section{INTRODUCTION}

Nowadays, it is generally acknowledged that one of the most common degradation mechanisms of polymer materials is oxidation. To prevent or slow down this phenomenon, producers usually introduce antioxidants (AOs) and UV stabilizers inside the polymer compounds. The two main kinds of AOs are free-radical scavengers and hydroperoxide decomposers. The former are also known as primary AOs (usually phenol and amine-based molecules) while the latter are often called secondary AOs (thioether and phosphitebased molecules).

Up to now, the impact of these additives on the chemical and thermal response of the polymer compound is broadly analyzed in materials engineering literature [1-4]. On the contrary, their effect on the electrical properties and their response over aging have been partially analyzed in the electrical engineering research.

As a matter of fact, electrical studies often focus on the macroscopic behavior of the material for the application under consideration but ignore the effects of damage and microstructural changes $[5,6]$.

In this context, this work aims at identifying a connection between the micro- and macroscopic properties of twisted pair cables for nuclear applications and the changes in their chemical composition during the radio-thermal aging.

To do so, primary insulations has been analyzed by means of both non-destructive electrical and physico-chemical techniques. Dielectric spectroscopy was used to characterize the changes in the electrical response with aging time, whereas Fourier Transform Infrared (FTIR) spectroscopy and measurement of the Oxidation Induction Time (OIT) allowed the detection of the residual antioxidants and traces of oxidation products caused by aging.

In the end, correlations between electrical properties and $\mathrm{AO}$ content have been carried out.

This research is part of the H2020 Euratom TeaM Cables Project which aims at providing nuclear power plants (NPPs) operators with a novel methodology for efficient and reliable NPP cable ageing management, through, inter alia, developing a new multiscale modelling approach to study polymer radiation aging.

\section{MATERIALS AND METHODS}

\section{A. Specimens}

Samples analyzed are low-voltage I\&C cables with XLPE insulation used in NPPs, especially designed for the project. The cables here analyzed have a twisted pair configuration, whose structure is reported in Figure 1. The twisted pair cable is made up of three pairs with three different primary insulations, due to page constraints only one kind of insulating material is investigated. This is made up of silane crosslinked polyethylene stabilized with 1 phr of primary AO (phenol-based) and 1 phr of secondary AO (thioether-based). Each cable specimen is about $50 \mathrm{~cm}$ long.

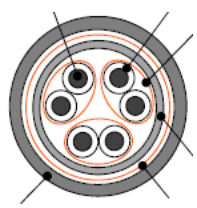

Fig. 1. Multilayer structure of twisted pair cables under investigation. (black) Conductor - Copper, (white) Primary insulation, (black) Shielding Copper wire braid, (grey) Sheath - Low smoke zero halogen

\section{B. Accelerated aging}

Three different dose rates, high, medium and low, corresponding to 400, 60 and $7 \mathrm{~Gy} / \mathrm{h}$ respectively, have been chosen to perform cable aging. Radio-thermal ageing was performed in the Panoza (Medium and Small Dose rate) and Roza (High Dose Rate) facilities at UJV Rez, Czech Republic, through a ${ }^{60} \mathrm{Co} \gamma$-ray source.

Aging conditions are summarized in Tab. 1. 
TABLE I. AGING CONDITIONS

\begin{tabular}{|l|c|c|c|}
\hline \multirow{2}{*}{$\begin{array}{c}\text { Aging } \\
\text { type }\end{array}$} & \multicolumn{3}{|c|}{ Aging properties } \\
\cline { 2 - 4 } & Dose rate $($ Gy/h) & Sampling time $(\boldsymbol{h})$ & $\begin{array}{c}\text { Max. absorbed } \\
\text { dose }(\boldsymbol{k G y})\end{array}$ \\
\hline Small & 7 & 3456 & 81 \\
\hline Medium & 60 & 864 & 286 \\
\hline High & 400 & 167 & 334 \\
\hline
\end{tabular}

At this stage, few samplings for the small dose rate (SDR) aging are present, so that SDR characterization is made up of a lower number of points.

\section{Dielectric spectroscopy measurements}

Complex permittivity and tan $\delta$ have been investigated through a Novocontrol Alpha dielectric analyzer with applied voltage of $3 \mathrm{~V}_{\text {rms }}$ in the frequency region $10^{6}-10^{-2} \mathrm{~Hz}$. Tests have been performed in oven at $50{ }^{\circ} \mathrm{C}$.

Tan $\delta$, known also as dissipation factor (DF), is defined by $[3,7,8]$ :

$$
\tan \delta=\frac{\omega \varepsilon^{\prime \prime}+\sigma}{\omega \varepsilon^{\prime}}
$$

where $\varepsilon$ ' is the real part of permittivity defined as the dielectric constant of the material, $\varepsilon$ "' is the imaginary part of permittivity related to the dielectric losses of the material, $\omega$ is the angular frequency of the wave and $\sigma$ is the DC conductivity of the material.

\section{Oxidation induction time (OIT) measurements}

OIT has been measured at $210{ }^{\circ} \mathrm{C}$ with a TA instrument DSC Q10 calorimeter. XLPE samples with a mass between 5 and $10 \mathrm{mg}$ has been inserted inside the instrumentation furnace in an open aluminium pan. First heating run from room temperature to $210{ }^{\circ} \mathrm{C}$ has been performed with an heating rate of $10^{\circ} \mathrm{C} \cdot \mathrm{min}^{-1}$ under a pure $\mathrm{N}_{2}$ flow $(50 \mathrm{~mL} \cdot \mathrm{min}$ $\left.{ }^{1}\right)$; then, temperature equilibrium has been set at $210^{\circ} \mathrm{C}$ for 5 min and finally, the gas has been switched from $\mathrm{N}_{2}$ to $\mathrm{O}_{2}$ for isotherm at $210^{\circ} \mathrm{C}$. Under pure oxygen, the test has been stopped once an exothermal peak, referred to the induction of the oxidation, has been registered. The time period passed between the switching of the furnace atmosphere and the onset of the exothermal peak (graphically obtained through the tangent method) is defined as OIT.

\section{E. Fourier Trasnform Infrared (FTIR) measurements}

FTIR spectra has been recorded in attenuated total reflectance (ATR) mode using a Perkin Elmer FTIR Frontier spectrometer equipped with a diamond/ZnSe crystal. Each spectrum is the average of 16 scans in the spectral range between 4000 and $650 \mathrm{~cm}^{-1}$, resolution has been set equal to $4 \mathrm{~cm}^{-1}$. Any change in absorbance has been normalized with the absorbance of $\mathrm{CH}_{2}$ scissoring vibrations of PE crystal phase at $1472 \mathrm{~cm}^{-1}$.

\section{EXPERIMENTAL RESULTS}

\section{A. Dielectric spectroscopy}

Figure 2 shows the trend of the dielectric losses $(\tan \delta)$ at $100 \mathrm{kHz}$ as a function of the total dose for the analyzed aging conditions.

As expected, the values of the dielectric losses raise with the increase of the aging period. From Figure 2, it is evident that the dose rates do have an impact on dielectric response of the material during aging. Different dose rates, indeed, result in different values of $\tan \delta$, for a fixed absorbed dose. This discrepancy is more and more evident as we increase the total dose (or the aging time). In particular, the higher the dose rate, the larger the dielectric losses. Indeed, as it will be discussed in the following, the severity of the environmental conditions, namely the $\gamma$-rays strength, can catalyze the radical formation which results into the increase of the dipolar species inside the polymer matrix (mainly big polar molecules e.g. AOs products and oxidized chains), resulting into the increase of the dissipation factor in the dipolar polarization frequency region (around $10^{5} \mathrm{~Hz}$ ).

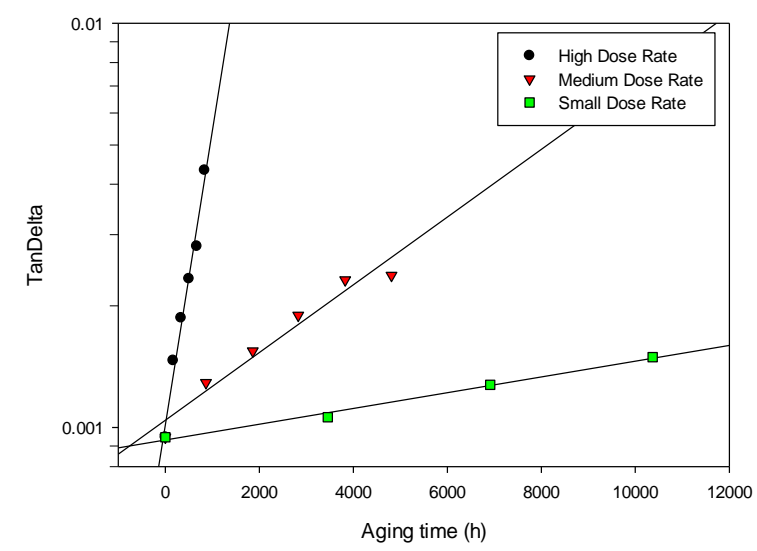

Fig. 2. Tan $\delta$ at $100 \mathrm{kHz}$ for twisted pair cable as a function of the aging time for the three different aging conditions considered. (green) Small Dose Rate, (red) Medium Dose Rate, (black) High Dose Rate.

\section{B. OIT results}

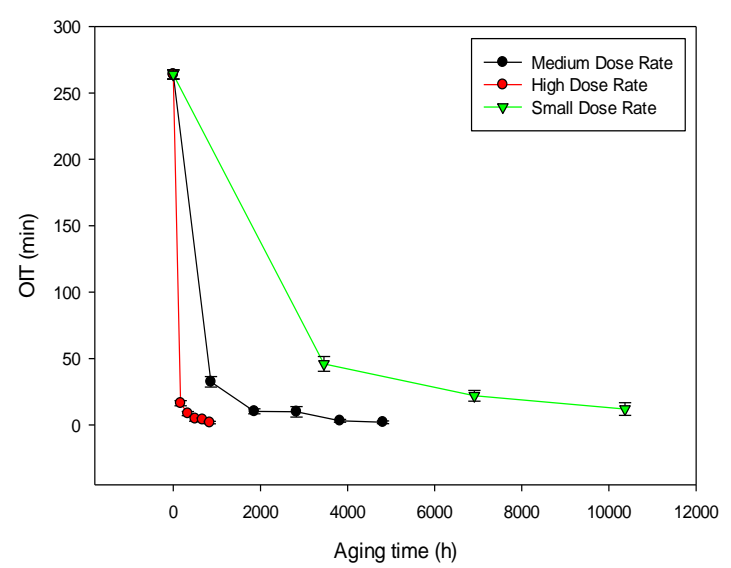

Fig. 3. OIT values for twisted pair cable as a function of aging time for the three different aging conditions considered. (green) Small Dose Rate, (red) Medium Dose Rate, (black) High Dose Rate.

Figure 3 reports the trend of the OIT values as a function of the aging time for the three aging conditions analyzed. OIT decreases with the increase of aging time, as expected. This is imputable to the gradual consumption of AOs due to the environmental stressors which catalyze oxidative reactions. In all the cases, the major reduction of the OIT values happens into the first aging period (reduction by a factor 6). Further aging causes the decreasing of OIT in a less drastic way, reaching finally a non-null value for all the conditions 
analyzed ( 2 mins). Even in this case, the higher the dose rate, the faster the decreasing of OIT, as supposed. It is worth commenting that, due to the graphical extrapolation of data, 2 minutes represents the minimum value recordable by the instrumentation; so that it is possible to claim that oxidation may have started even if the OIT owns a non-zero value $(2$ mins).

\section{FTIR results}
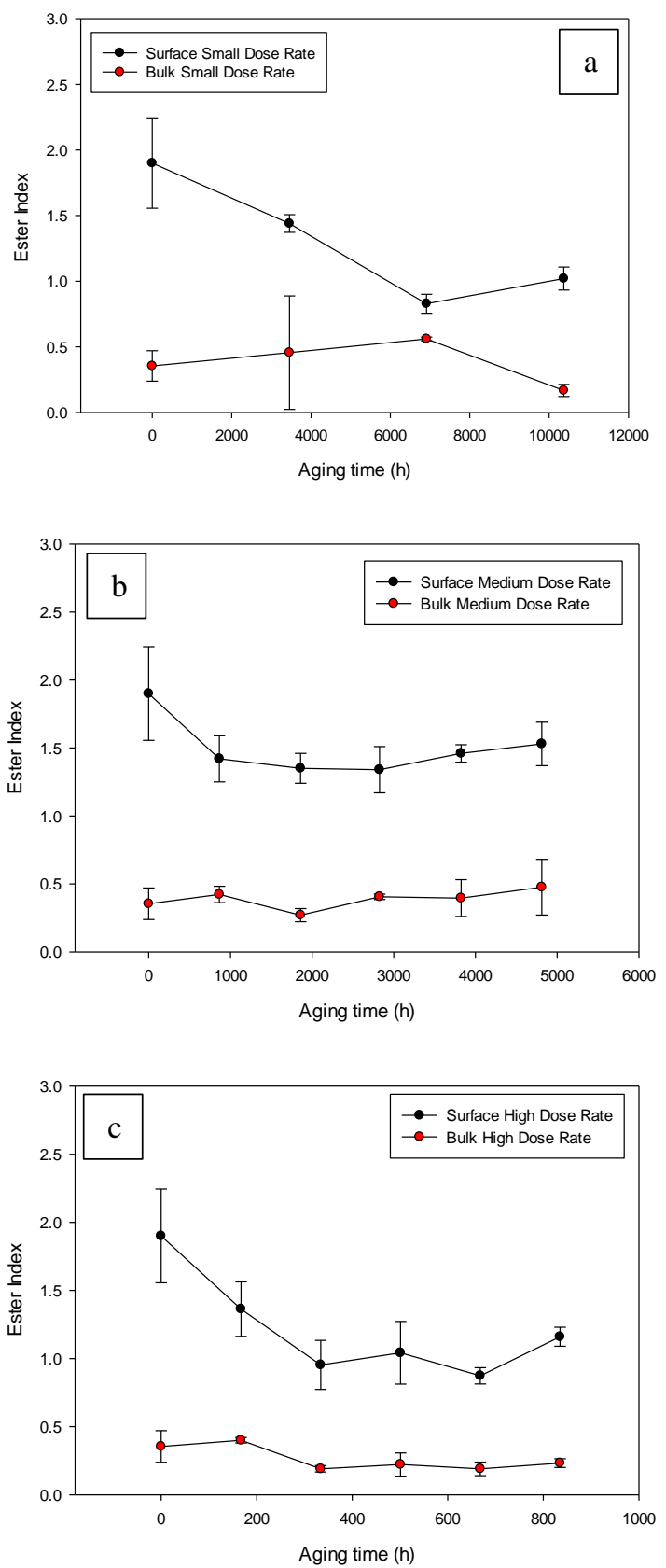

Fig. 4. Ester index for twisted pair cable as a function of aging time for the three different aging conditions considered. (a) Small Dose Rate, (b) Medium Dose Rate, (c) High Dose Rate.

Figure 4 displays the behavior of ester index (absorbance ratio between the $1733 \mathrm{~cm}^{-1}$ ester peak and the reference peak at $1472 \mathrm{~cm}^{-1}$ ) with aging time in the bulk (red dots) and on the outer surface (black dots) of the insulation, for the three aging conditions considered.
In all the cases analyzed, an initial decrease of the ester index (EI) is shown on the surface of the material. The reduction of the EI lasts for the initial two (small and high dose rate) and one (medium dose rate) aging period, claiming a dependence on both the aging time and the aging severity (dose rate). Since the primary AO owns ester bonds itself, the ester index can be linked to the concentration of AOs on the surface. Indeed, AOs cannot be properly dispersed in the amorphous region of the XLPE matrix due to their high concentration (1 phr) and they are thus also present under the form of crystalline nodules on the insulation surface [3]. Consequently, the initial reduction of EI can be likely imputed to the consumption of both well dissolved and crystallized AOs on the surface during the first aging periods.

After the initial decrease, in all the cases shown one can observe a stabilization of the EI on the surface followed by a slightly increase of the EI, likely associated with an initial oxidative state of the polymer surface.

In the bulk, the variation of the EI is almost negligible suggesting a very weak modification of the ester content, which is mainly occurring on the surface, as stated above.

It is worth commenting that also, in this case, the dose rate plays a significant role. Indeed, the duration of the decreasing phase is different among the three aging conditions due to the gamma ray intensity.

\section{DISCUSSION}

It has been shown that radiochemical aging causes a deep modification of the chemical and electrical properties of the cable specimens analyzed. In brief, this behavior could be imputed to the severity of the aging conditions ( $\gamma$-rays) which is reported in literature $[1,3,4]$ to accelerate degradation phenomena, i.e. consumption and physical loss of AOs. This mechanism leads to oxidation and, consequently chain scission and chemi-crystallization (main causes of the loss of mechanical performance of the polymer matrix).

It is evident that the main cause of degradation of the polymeric insulation is oxidation which is usually avoided by the presence of AOs. These molecules, as presented before, are highly polar species and convert themselves into other polar species reacting with oxygen atoms, namely AOs products. The consumption of the AOs causes the reduction of the OIT values and the formation of new molecules. These molecules show the same ester bond so that the two phenomena (AO consumption and AO products formation) can be difficulty singled out through FTIR measurements (Figure 4) due to possible compensation of concentration of the ester bonds leading to the flattening of the EI trend with aging. As presented in the previous section, the last aging times for the three aging conditions show an increase of the EI which is also linked to the minimum value reachable by the OIT measurement (Figure 3), possibly suggesting that oxidation has started and the new esters created are directly referred to the polymer oxidized species.

Focusing on the electrical behavior of the aging treatment (Figure 2), as presented, one can observe the increase of the dielectric losses with aging time, as the AOs products do. These latter are more easily analyzed through the dielectric spectroscopy technique. AO molecules, indeed, react with radical chains giving birth to bigger molecules (e.g. hydroperoxide and AO-grafted polymer chains) which can respond electrically in the frequency region here analyzed. 
For this reason, the conversion of the AOs into their products may be directly linked to the increase of the $10^{5} \mathrm{~Hz}$ dielectric losses and, consequently, to the aging of the polymer matrix.

Let us introduce the Antioxidants Depletion Factor (ADF) defined as:

$$
\mathrm{ADF}=\frac{\mathrm{OIT}_{0}-\mathrm{OIT}}{\mathrm{OIT}_{0}}
$$

where OIT and $\mathrm{OIT}_{0}$ are the respective values for the aged and unaged samples.

As the name suggests, this factor represents the depletion of the AOs over the aging time, hence their conversion into AO products. Indeed, OIT values are directly proportional to the AO concentration as widely reported in literature [3, 9].

Figure 5 reports the trend of the dielectric losses as a function of the defined ADF.

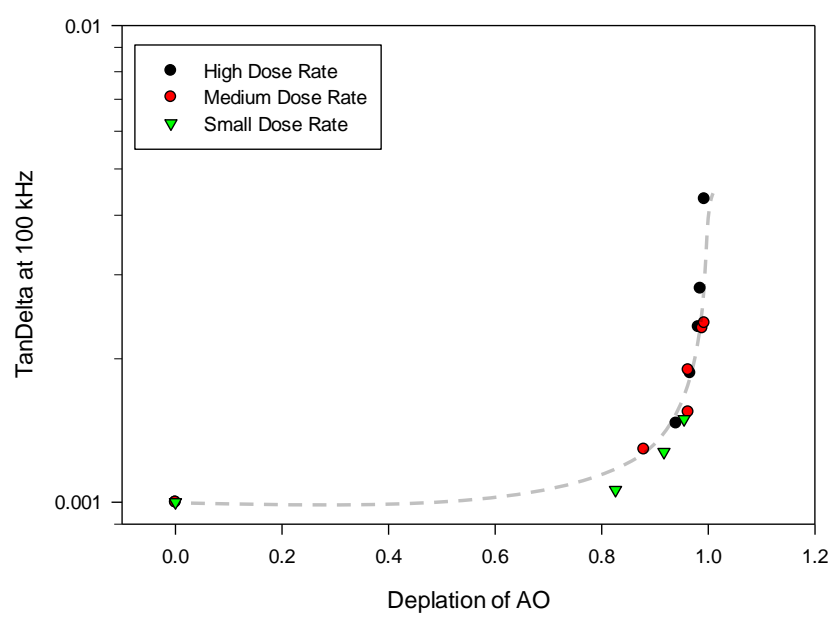

Fig. 5. Depletion of AO as a function of TanDelta for twisted pair cable and the three different aging conditions considered. (green) Small Dose Rate, (red) Medium Dose Rate, (black) High Dose Rate.

The association with dielectric losses lays on the fact that both the factors keep into account the contribution of the changes of AO concentration. The dielectric spectroscopy at the analyzed frequencies (around $10^{5} \mathrm{~Hz}$ ), indeed, may evaluate the presence and the concentration of the $\mathrm{AO}$ products (dipolar species). On the other side, as stated above, OIT is directly dependent on the concentration of AOs inside the polymer matrix [3, 9], hence it is able to evaluate the conversion of these molecules into their products.

For all the aging conditions considered, after a significant initial variation of $\mathrm{ADF}$, the data are placed between the range $0.8-1$ tending asymptotically to 1 , corresponding to the complete running out of AOs (namely when OIT $=0$ ).

This correlation is possible until the AOs are present, hence, when they are fully converted into AO products and no oxidation occurs, so that OIT $\neq 0$.

As a matter of fact, various works correlate the increase of the ester index after the AO consumption to the increase of the dielectric losses at high frequencies [3]. Indeed, the same chemical species have their electrical response placed in the region around $10^{5} \mathrm{~Hz}$.

In the case here considered, one can observe a slightly increase of the EI and an OIT close to zero only during the last aging period considered for the three aging conditions. Therefore, the actual aging level seems to be not sufficient to propose correlations between these two parameters at this stage.

\section{CONCLUSIONS}

In this work, different aging assessment techniques have been reported to efficiently evaluate the aging-induced modification of the polymer insulation of the cable analyzed. In particular, chemical analyses, i.e. OIT and FTIR, have been linked to the change in the electrical response of the material, underlining a quite good correlation among the techniques. The introduction of the antioxidant depletion factor and consequently, the grade of the AO conversion into products induced by aging stressors, allowed the linking of this property with the dissipation factor $(\tan \delta)$ at $100 \mathrm{kHz}$. This latter resulted to be strictly related to the concentration of the antioxidant products, leading thus to an effective correlation between the two factors.

Future works on this topic will include the analysis of the effect of the radiochemical aging on the different cable configurations (e.g. coaxial cable) and the chemical composition (e.g. introduction of inorganic fillers) of the insulation system.

\section{ACKNOWLEDGMENT}

The project leading to this application has received funding from the

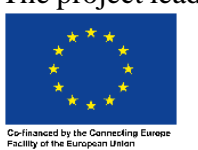
Euratom research and training programme 20142018 under grant agreement No 755183. The authors are grateful to Nexans and UJV for sample preparation and aging.

\section{REFERENCES}

[1] N. Khelidj, X. Colin, L. Audouin, J. Verdu, C. Monchy-Leroy, and V. Prunier, "Oxidation of polyethylene under irradiation at low temperature and low dose rate. Part II. Low temperature thermal oxidation," Polymer Degradation and Stability, vol. 91, no. 7, pp. 1598-1605, Jul. 2006, doi: 10.1016/j.polymdegradstab.2005.09.012.

[2] V. Plaček, B. Bartoníček, V. Hnát, and B. Otáhal, "Dose rate effects in radiation degradation of polymer-based cable materials," Nuclear Instruments and Methods in Physics Research Section B: Beam Interactions with Materials and Atoms, vol. 208, pp. 448-453, Aug. 2003, doi: 10.1016/S0168-583X(03)00626-8.

[3] S. V. Suraci, D. Fabiani, A. Xu, S. Roland, and X. Colin, "Ageing Assessment of XLPE LV Cables for Nuclear Applications Through Physico-Chemical and Electrical Measurements," IEEE Access, vol. 8, pp. 27086-27096, 2020, doi: 10.1109/ACCESS.2020.2970833.

[4] B. Fayolle, E. Richaud, X. Colin, and J. Verdu, "Review: degradationinduced embrittlement in semi-crystalline polymers having their amorphous phase in rubbery state," $J$ Mater Sci, vol. 43, no. 22, pp. 6999-7012, Nov. 2008, doi: 10.1007/s10853-008-3005-3.

[5] S. W. Glass, A. M. Jones, L. S. Fifield, and T. S. Hartman, "Frequency domain reflectometry NDE for aging cables in nuclear power plants," presented at the 43RD ANNUAL REVIEW OF PROGRESS IN QUANTITATIVE NONDESTRUCTIVE EVALUATION, VOLUME 36, Atlanta, Georgia, USA, 2017, p. 080015, doi: 10.1063/1.4974640.

[6] N. Bowler and S. Liu, "Aging Mechanisms and Monitoring of Cable Polymers," Int, J, Prognostics and Health Management, vol. 6, 2015.

[7] S. V. Suraci, D. Fabiani, K. Sipilä, and H. Joki, "Filler impact analysis on aging of crosslinked polyethylene for nuclear applications through dielectric spectroscopy," p. 4.

[8] S. V. Suraci, D. Fabiani, L. Mazzocchetti, and L. Giorgini, "Degradation Assessment of Polyethylene-Based Material Through Electrical and Chemical-Physical Analyses," Energies, vol. 13, no. 3, p. 650, Jan. 2020, doi: 10.3390/en13030650.

[9] S. Liu, S. W. Veysey, L. S. Fifield, and N. Bowler, "Quantitative analysis of changes in antioxidant in crosslinked polyethylene (XLPE) cable insulation material exposed to heat and gamma radiation," Polymer Degradation and Stability, vol. 156, pp. 252-258, Oct. 2018, doi: 10.1016/j.polymdegradstab.2018.09.011. 